\title{
Efficacy Of Three Different Regimens In Eradication Of Helicobacter Pylori Infection In Nepalese Patients of Gandaki Region
}

\author{
Subash Bhattarai ${ }^{1 *}$, Chandra Prasad Acharya ${ }^{2}$, Sudeep Regmi ${ }^{3}$ \\ ${ }^{1,2}$ Department of Medicine, ${ }^{3}$ Department of Pathology, Manipal College of Medical Sciences, Pokhara, Nepal
}

\begin{abstract}
BACKGROUND

This work is licensed under a Creative Commons Attribution 4.0 Unported License.

Helicobacter pylori ( $\mathrm{H}$. pylori) is associated with the pathogenicity of gastro-duodenal ulcers and gastric cancers. Combination of several antimicrobial therapies and regimens have been advised for $\mathrm{H}$. pylori treatment. But, resistance to various antibiotics regimens are being documented worldwide. The present study was undertaken to study the efficacy of commonly used 3 different regimens for eradication of H.pylori infection in Nepalese patients.
\end{abstract}

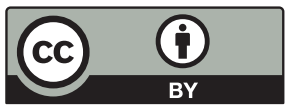

\section{METHODS}

A cross-sectional, hospital based study comprising of 405 subjects, was conducted. Each study patient underwent upper gastro-intestinal endoscopy followed by rapid urease test or histopathology from the biopsy sample for $\mathrm{H}$. pylori detection. Patients were randomly subjected to 3 different $\mathrm{H}$. pylori eradication regimen. After 4 weeks of therapy, patients were reevaluated for persistence of H. pylori infection either by repeat UGI endoscopy followed by RUT or C14- Urea Breadth Test. Data analysis was done by SPSS 20.

\section{RESULTS}

Mean age of the patients was $34.4 \pm 8.72$ years ( $M: F=1.5: 1$ ) with male predominance. H. pylori eradication rate was only $65.9 \%$ in patients using standard triple regimen using Amoxicillin, Clarithromycin and PPI (Group A). Eradication rate was greater (77.8\%) with Levofloxacin based regimen (Group B) and 83.3\%. with sequential regimen containing Amoxicillin followed by Clarithromycin and Tinidazole with PPI (Group C). Conclusion: The study demonstrates that the current standard Amoxicillin and Claritrhromycin based triple regimen has lowest eradication rate followed by levofloxacin based regimen. The sequential regimen was the most efficacious among the 3 different regimens for $\mathrm{H}$. pylori eradication.

\section{KEYWORDS}

antibiotics; eradication; Helicobacter pylori; rapid urease test; urea breadth test

\section{INTRODUCTION}

Helicobacter pylori ( $\mathrm{H}$. pylori) is a very common human infection. Approximately, $50 \%$ of world population harbor H. pylori, though only $10-20 \%$ are symptomatic. ${ }^{3}$ H. pylori infection has an important role to play in pathogenicity of gastric and duodenal ulcers. A strong evidence exist between risk of gastric cancer, gastric mucosa associated lymphoid tissue lymphomas and $\mathrm{H}$. pylori infection. ${ }^{2} \mathrm{H}$. pylori infections that involves the antrum predominantly predispose to duodenal ulceration whereas intense inflammation in the oxyntic mucosa will result in gastric atrophy with a decreased acid output and a predisposition to gastric ulceration and cancer. ${ }^{3}$

Upper gastrointestinal (UGI) endoscopy is the modality of choice for evaluation of UGI tract disorders including dyspepsia. Various non-invasive and invasive tests aided by UGI endoscopy are available for detection of $\mathrm{H}$. pylori.
Several combination therapies have been an effective standard of treatment. ${ }^{4}$ The standard treatment for $\mathrm{H}$. pylori infection worldwide and also practiced in Nepal are two antibiotics like Clarithromycin, Amoxicillin or Metronidazole in conjunction with proton pump inhibitors (PPI). Resistance to all these antibiotics and various regimens are being documented worldwide.5,6 Similar data and studies regarding efficacies of different regimens for eradication of $H$. pylori infection and their resistance patterns in patients with dyspepsia from this part of the country are scanty. The present study was undertaken to study the efficacy of commonly used 3 different regimens for eradication of H.pylori infection in Nepalese patients with dyspepsia attending a tertiary care Teaching Hospital of Gandaki region.

*Corresponding Author | Dr Subash Bhattarai, Department of Medicine,

Manipal College of Medical Sciences, Pokhara, Nepal

Email: kiwisubash@yahoo.com 


\section{METHODS}

This observational, cross-sectional, prospective hospital based study was carried out in the department of Medical Gastroenterology under department of Medicine at Manipal College of Medical Sciences and Teaching Hospital, Nepal from January 2019 to November 2020. The sample size was collected using the formula:

Sample size: $Z^{2} \times[p \times(1-p)] / e^{2}$

Z: 1.96 (critical value of the normal distribution for 95\% confidence interval)

'p: sample proportion (prevalence of the disease or 0.5 if no prevalence is known)

e: standard error (0.05) or when prevalence is given, $20 \%$ of prevalence

The minimum sample size required and calculated as per the equation with no known prevalence of $\mathrm{H}$ pylori in dyspepsia; $95 \% \mathrm{Cl}(\mathrm{Z}=1.96$, e=0.05, and assumed $\mathrm{p}=0.5$, $q=0.5)$ was 384 .

All consecutive patients aged more than 18 years, either attending OPD or admitted in ward who underwent Upper Gastrointestinal (UGI) endoscopy for dyspepsia and various upper GI symptoms from January 2019 till November 2020 were enrolled for the study. Patients with use of recent $H$. pylori eradication therapy or use of any antibiotics or proton pump inhibitors in last 2 weeks and those with upper GI malignancy and those with incomplete records were excluded from the study.

A detailed clinical history, relevant physical and abdominal examinations was carried out. Each patient underwent endoscopic investigation by standard flexible gastro duodenal endoscope (PENTAX EPK 700, PENTAX JAPAN Inc.) and diagnostic findings were documented. Endoscopic biopsies were taken from the antrum close to the pylorus and corpus both. Each specimen underwent rapid urease test of biopsy specimen. After biopsy, the tissue was inserted in a commercially available Rapid Urease Test (RUT) kit (GASTRO CURE system, KOLKOTA, WEST BENGAL ). The test was performed at the time of gastroscopy. A drop of distilled water was placed into the medium containing urea and an indicator, phenol red within the kit. The urease produced by $\mathrm{H}$. pylori hydrolyzes urea to ammonia, and changes the color of the kit from yellow (negative) to red or pink (positive). Most turn positive within 120 to 180 minutes but it is best to be read up to 24 hours. Selective biopsies with endoscopic findings like mucosal disease and ulcers underwent Hematoxylin and Eosin (H\&E) staining for detection of $\mathrm{H}$ pylori with histopathology assessment in the department of Pathology by consultant pathologist. $H$. pylori infection was diagnosed if one or both test results were positive

All the consecutive subjects were randomly allocated to three different Groups/Treatment Regimens.

Standard triple therapy (Group A)

Amoxicillin $1 \mathrm{~g}$ (twice daily) + Clarithromycin 500 mg (twice daily) + PPI : Pantoprazole 40 mg (twice daily). Total duration of treatment (14 Days).

Levofloxacin based (Group B)

Amoxicillin (1 g twice daily) + Levofloxacin (500 mg once dail ) + Pantoprazole (40 mg twice daily) for 10 days

Sequential therapy (Group C)

(DAY 1-5): Amoxicillin (1 g twice daily) + Pantoprazole (40 mg twice daily)

(DAY 6-10): Clarithromycin 500 mg (twice daily) + Tinidazole 500 mg ( twice daily) + Pantoprazole 40 mg (twice daily)

Efficacy of different $\mathrm{H}$ pylori eradication regimens were assessed with C 14-Urea Breadth Test (Shenzhen Zhonghe Headway Bio-Sci \& Tech CO. Ltd China) at least 4 weeks after the end of the antimicrobial therapy. Patients were asked to swallow a ${ }^{14} \mathrm{C}$-labelled Urea capsule (tracer) with about $200 \mathrm{ml}$ of water. Urease produced in excessive quantity by $\mathrm{H}$. Pylori hydrolyze the Urea and produces ${ }^{14} \mathrm{C}$-labelled $\mathrm{CO}^{2}$. Exhaled ${ }^{14} \mathrm{CO}^{2}$ was collected, analyzed and detected by the detecting device showing positive or negative results. Compliant patients with erosive mucosal diseases or ulcers were otherwise subjected to repeat UGI endoscopy followed by RUT for $\mathrm{H}$. pylori detection after 4 weeks of antimicrobial therapy.

This study was approved by the Institutional Review Board of Manipal College of Medical sciences (IRB number: MEMG/IRC/413/GA). Patients or their relative's informed consent were taken for participation in the study. Data were collected in a pre-structured proforma. All categorical data were expressed in absolute number and percentage. All numerical continuous data were expressed in mean $\pm S D$. The data analysis was done using Statistical Packages for the Social Sciences 20. (IBM Corp. Released 2011. IBM SPSS Statistics for Windows, Version 20.0. Armonk, NY: IBM Corp.).

\section{RESULTS}

One Thousand one Hundred and forty patients underwent UGI endoscopy followed by $H$. pylori detection by RUT or histopathology or both during the study period. H. pylori was detected in 624 patients showing a prevalence of $54.7 \%$ among patients undergoing UGI endoscopies for dyspepsia. Rapid urease test was positive for $\mathrm{H}$. pylori in 604 patients. 
Histopathology detected $\mathrm{H}$. pylori by $\mathrm{H} \&$ E staining in only 42 (28.4\%) patients among 148 biopsies. But among these 624 patients with evidence of $\mathrm{H}$. pylori, 163 lost to follow ups and 56 could not tolerate the regimens. Only 405 subjects underwent repeat testing for $\mathrm{H}$. pylori after complete use of $\mathrm{H}$. pylori treatment regimens. The total number of final study subjects was hence only 405.

There were 244 males and 161 females (M: $F=1.5: 1$ ). Mean age of the patients was $34.4 \pm 8.72$ years (range of 18 to 88 years). Esophagogastroduodenoscopy (EGD) was normal in 85 study subjects. Rest subjects had findings as depicted in Table 1.

Table 1: UGI endoscopic findings among study subjects

\begin{tabular}{|l|r|r|}
\hline UGI endoscopy findings & Number $(\mathbf{n})$ & Percentage (\%) \\
\hline Gastric ulcers & 45 & 11.1 \\
\hline Duodenal ulcers & 63 & 15.5 \\
\hline Erosive mucosal disease & 191 & 47.2 \\
\hline Others & 21 & 5.2 \\
\hline Normal Findings & 85 & 21 \\
\hline Total & $\mathbf{4 0 5}$ & $\mathbf{1 0 0}$ \\
\hline
\end{tabular}

Among these 405 patients, maximum of 255 received the standard triple regimen containing amoxicillin, clarithromycin and PPI (Group A). Group B included 90 subjects and received Levofloxacin based regimen. Rest 60 patients received sequential therapy containing Amoxicillin followed by Clarithromycin and Tinidazole with PPI (Group C). After completion of $\mathrm{H}$. pylori therapy, they were subjected to repeat $\mathrm{H}$. pylori testing as in Table 2. Only 189 patients underwent repeat UGI endoscopy followed by RUT. The rest 216 subjects were tested with UBT.

Table 2: H. pylori detection before and after treatment among different study sub groups

\begin{tabular}{|c|c|c|c|c|}
\hline \multirow{2}{*}{\multicolumn{2}{|c|}{ H. pylori detection before treatment }} & \multirow{2}{*}{$\begin{array}{c}\text { Group A } \\
255\end{array}$} & \multirow{2}{*}{$\frac{\text { Group B }}{90}$} & \multirow{2}{*}{$\frac{\text { Group C }}{60}$} \\
\hline & & & & \\
\hline \multirow{2}{*}{ Urea Breath test after treatment } & Positive & 48 & 11 & 5 \\
\hline & Negative & 87 & 37 & 28 \\
\hline \multirow{2}{*}{ Rapid Urease Test after treatment } & Positive & 39 & 9 & 5 \\
\hline & Negative & 81 & 33 & 22 \\
\hline \multicolumn{2}{|l|}{ Eradication success } & $168(65.9 \%)$ & $70(77.8 \%)$ & $50(83.3 \%)$ \\
\hline
\end{tabular}

H. pylori eradication rate was only $65.9 \%$ in patients using standard triple regimen using Amoxicillin, Clarithromycin and PPI (Group A). Eradication rate was greater (77.8\%) with Levofloxacin based regimen (Group B). Sequential therapy containing Amoxicillin followed by Clarithromycin and Tinidazole with PPI (Group C) had the maximum eradication rate of $83.3 \%$.

\section{DISCUSSIONS}

Four hundred and five patients were enrolled in this study with 244 male and 161 female (M: $F=1.5: 1)$. Studies by Muzaffar et $\mathrm{al}^{7}{ }^{7}$ Uygun et $\mathrm{al}^{8}$ and Chang et $\mathrm{al}^{9}$ have also highlighted male predominance in their studies. Mean age of the patients was $34.4 \pm 8.72$ years in the current study. It was higher ( $44.3 \pm 15$ years) in the study by Muzaffar et al. ${ }^{7}$

The treatment purpose of $\mathrm{H}$. Pylori infection in any clinical situation is eradication of bacterium from the stomach. The main reasons for $\mathrm{H}$. pylori eradication failure are poor compliance, antibiotic resistance and may also be contributed due to diarrhoea secondary to antibiotics therapy. ${ }^{10}$

H. pylori eradication rate was only $65.9 \%$ in patients using standard triple regimen using Amoxicillin, Clarithromycin and PPI in the current study. Eradication rate was greater (77.8\%) with Levofloxacin based regimen. Sequential therapy containing Amoxicillin followed by Clarithromycin and Tinidazole with PPI had the maximum eradication rate of $83.3 \%$ in the current study. The eradication rate of H. pylori was $63 \%$ and $64.3 \%$ in the standard triple therapy group, and $80.1 \%$ and $81.9 \%$ in the sequential therapy group by Uygun et al.8 and Chang et al $^{9}$ respectively, findings almost similar to our study. The eradication rates were lower, $58.7 \%$ in the conventional triple therapy group and $75.9 \%$ in the sequential therapy group by Chung et al. ${ }^{11}$ Higher eradication rate up to $93.4 \%$ by sequential therapy was observed by Jafri et al. ${ }^{12}$ All these studies including studies by Choi et $\mathrm{al}^{13}{ }^{3}$, Sanchez-Delgado et $\mathrm{al}^{14}$, and Vaira et $\mathrm{al}^{15}$ have reported higher eradication rate with sequential therapy than standard triple therapy. In contrary to all these studies, Mehmet et al ${ }^{16}$ reported that sequential eradication regimen was not superior to standard triple regimen as a first-line therapy for $\mathrm{H}$. pylori eradication.
Eradication rate was $77.8 \%$ with Levofloxacin based regimen in the current study. It was $81 \%$ according to Muzaffar et al. ${ }^{7}$ It was even higher; $92 \%$ and $92.5 \%$ according to studies by Rakici et $\mathrm{al}^{17}$ and Dib et al ${ }^{18}$ respectively. All these studies have highlighted better $\mathrm{H}$. pylori eradication with levofloxacin based regimens when compared with the conventional triple regimen comprising of Amoxicillin, Clarithromycin and PPI. 
Muzaffar et $\mathrm{al}^{7}$ achieved least eradication (68\%) by standard triple regimen, $81 \%$ by Levofloxacin based regimen and maximum eradication (86\%) by sequential regimen respectively. These findings were in consistency with the present study.

\section{CONCLUSIONS}

The study demonstrates that the current standard Amoxicillin and Clarithromycin based triple regimen has low eradication rate. The Levofloxacin based regimen was more effective than conventional therapy. The sequential regimen comprising of Amoxicillin followed by Clarithromycin and Tinidazole with PPI had even more $\mathrm{H}$. pylori eradication rate and was the most efficacious among the 3 different regimens.

\section{LIMITATIONS OF THE STUDY}

This was the study of population of Gandaki region and may not be representative of the entire national populations. Individual resistance to the antibiotics Amoxicillin, Clarithromycin, Levofloxacin and Tinidazole has not been studied. Side effects and patients compliance to each regimen was also not studied.

\section{CONFLICT OF INTEREST: NONE}

\section{SOURCE OF FUNDING: NONE}

\section{REFERENCES}

1. Go MF. Natural history and epidemiology of Helicobacter pylori infection. Aliment Pharmacol Ther. 2002; 16(1):3-15.

2. Fauci AS, Braunwald E, Kasper DL, et al. (2008). H Pylori the bacterium. Harrison principles of internal medicine 17th Edn Pp: 1858.

3. Suerbaum S, Michetti P. Helicobacter pylori infection. N Engl J Med. 2002; 347: 1175-86.

4. Vakil N. Helicobacter pylori treatment: a practical approach. Am J Gastroenterol. 2006; 101(3): 497-9.

5. Leal-Herrera $Y$, Torres J, Monath TP, et al. High rates of recurrence and of transient reinfections of Helicobacter pylori in a population with high prevalence of infection. Am J Gastroenterol. 2003: 98(11): 2395-2402.

6. Soto G, Bautista CT, Roth DE, et al. Helicobacter pylori reinfection is common in peruvian adults after antibiotic eradication therapy. J Infect Dis. 2003; 188(9): 1263-75.

7. Muzaffar M, Mir MM, Sumji S, et al. Sequential Therapy versus Standard Triple Therapy in Helicobacter pylori Eradication. G J Dig Dis. 2018; 4(3):1-8

8. Uygun A, Kadayifci A, Yesilora Z, et al. (2008) Comparison of sequential and standard triple-drug regimen for Helicobacter pylori eradication: a 14-day, open-label, randomized, prospective, parallel-arm study in adult patients with nonulcer dyspepsia. Clin Ther 30(3): 528-34.
9. Chang JY, Shim KN, Tae $\mathrm{CH}$, et al. Triple therapy versus sequential therapy for the first-line Helicobacter pylori eradication.. BMC Gastroenterology. 2017; 17(16):1-8.

10. Laine L, Fennerty MB, Osato M, et al. Esomeprazole based $H$. pylori eradication therapy and the effect of antibiotic resistance: results of three multicenter, double blind trial. Am J Gastroenterol. 2000; 95(12): 3393-8

11. Chung JW, Jung YK, Kim YJ, et al. Ten day sequential versus triple therapy for Helicobacter pylori eradication: A prospective, open label, randomized trial. J Gastroenterol Hepatol. 2012; 27(11):1675-80

12. Jafri NS, Honung CA, Houden CW. Meta-analysis Sequential therapy appears superior to standard therapy for H.pylori infections in patients naïve to treatment. Ann Intern Med.2008; 148(12): 923-31.

13. Choi WH, Park DI, Oh SJ, et al. Effectiveness of 10 daysequential therapy for Helicobacter pylori eradication in Korea. Korean J Gastroenterol.2008; 51(5): 280-4.

14. Sanchez-Delgado J, Calvet $X$, Bujande $L$, et al. Ten-day sequential treatment for Helicobacter pylori eradication in clinical practice. Am J Gastroenterol. 2008; 103(9): 2220-3.

15. Vaira D, Zullo D, Vakil N, et al. Sequential therapy versus standard triple therapy for Helicobacter pylori eradication: a randomized trial. Ann Intern Med. 2007; 146(8): 556-63.

16. Mehmet D, Hilmi A. The effects of sequential treatment as a first-line therapy for Helicobacter pylori eradication. Turk J Med Sci 2011; 41 (3): 427-33

17. Rakici H, Ayaz T, Akdogan RA, et al. Comparison of levofloxacinand moxifloxacin-based triple therapies with standard treatment in eradication of Helicobacter pylori as first-line therapy. Digestion. 2014; 90(4): 261-4.

18. Dib J Jr, Alvarez B, Mendez L, et al. Efficacy of PPI, levofloxacin and amoxicillin in the eradication of Helicobacter pylori compared to conventional triple therapy at a Venezuelan hospital. Arab J Gastroenterol. 2013; 14(3):123-5. 